\title{
HIGH-RISE AIRFLOW STRUCTURAL CONCEPT
}

\author{
M.C. PHOCAS, O. KONTOVOURKIS \& N.I. GEORGIOU \\ Department of Architecture, University of Cyprus, Cyprus.
}

\begin{abstract}
Energy technologies realized through kinetic mechanisms in high-rise buildings may maximize the performance and sustainability of the buildings and their urban environments. In search for innovation through respective technological advancements, the interactive design of the building form and its structural components is significant. At the same time, the superposition of the living organisms' vocabulary on the built environment delivers new insights and innovative solutions for sustainable developments through the integral composition of the components, light-weight and kinetic behaviour of the structures. In this frame, biomimetic-driven research and application leads to new architectural design concepts. Along these lines, the current paper exemplifies the design, simulation and analysis of a high-rise hybrid structure of $250 \mathrm{~m}$ height and $25 \mathrm{~m}$ diameter, which has an innovative lightweight load-bearing system and incorporates an integrated kinetic core mechanism for providing through vertical airflow, improvement of the environmental conditions for the building spaces and the surrounding urban areas of high density. The kinetic mechanism is envisioned to operate as an urban ventilation chimney for air polluted cities and contribute to microclimate improvements. Through presentation of the high-rise airflow structural system, significant influencing factors and interdependencies towards sustainable, integrated biomimetic-driven solutions of high-rise structures with integrated kinetic subsystems for improved functionality will be discussed.
\end{abstract}

Keywords: bending-active members, high-rise buildings, hybrid systems, kinetic structures.

\section{INTRODUCTION}

Whereas in 1950, 30\% of the world population lived in urban areas, in 2000, the proportion of urban dwellers climbed to $47 \%$ and by the year 2030, it is projected to further rise to $60 \%$ with 5 billion people [1]. As a consequence of contemporary urban density, as well as consumerism and excessive use of energy sources, global warming, energy shortage, urban sprawl and air pollution comprise among other, significant factors, influencing negatively the sustainable development of urban centres. In this frame, high-rise buildings need to be redefined, in terms of achieving environmentally conscious, energy-saving and sustainable buildings through implementation of responsive materials and systems.

Sustainability through bioclimatic performance is primarily sought after the external building form. Wind flow uses scooping and 'Venturi' effect for air intake and exiting. Use can also be made for the pressure differential between the 'windward' and 'leeward' sides of the building for acceleration through ventilation and surface wind flow. Devices for wind flow intake and exiting can be adjustable and remotely controlled. They may be mounted on vertical, inclined, or horizontal surfaces. In addition, sky gardens and atria are common elements in support of internal airflow generation mechanisms. Furthermore, wind-energizers tunnel accelerated wind flow to activate generators. They usually have a closed climate system and smooth facades to ease the wind flow. Representative example for providing airflow through 
the building, is a proposal of a tall building with an integrated solar chimney presented in [2]. The basic operating principle is that air within a glass covered solar collector of a central chimney is heated, resulting in a warm and buoyant airflow through the collector and up the chimney. The airflow drives a turbine mounted at the base of the chimney.

Further alternatives in the generation of energy using non-polluting natural, renewable recourses in tall buildings, comprise applications of wind turbines of horizontal and vertical axis types [3]. These may be mounted on top, the sites, or integrated into the building cluster. A recent proposal for application of large-scale wind turbines was made for the Bahrain World Trade Center [4]. The two 50-storey sail-shaped office towers have a height of $240 \mathrm{~m}$ and support three $29 \mathrm{~m}$ diameter horizontal axis wind turbines. Accordingly, the building form and orientation are developed interactively with the overall energy concept. Wind power in buildings is seen as an opportunity in urban areas, but the built environment has relatively lower average wind speeds and higher turbulence levels. However, the disturbed flows around buildings can locally increase wind speeds and the energy yields may be increased compared to open sites [5]. On the other side, this technique faces deficiencies due to the often required turbines size and the fact that large turbines generate significant localized loads on the host building connection zones. In addition, potential environmental factors such as noise and vibration may become apparent, especially when the generators need to be large enough to require a gearing mechanism [6].

In view of the above, the current paper seeks to investigate an alternative redefinition of high-rise building structures that can be incorporated in dense urban environments and improve urban air ventilation through an incorporated secondary kinetic mechanism. The design proposal results from the integrative development of the structural elements that have

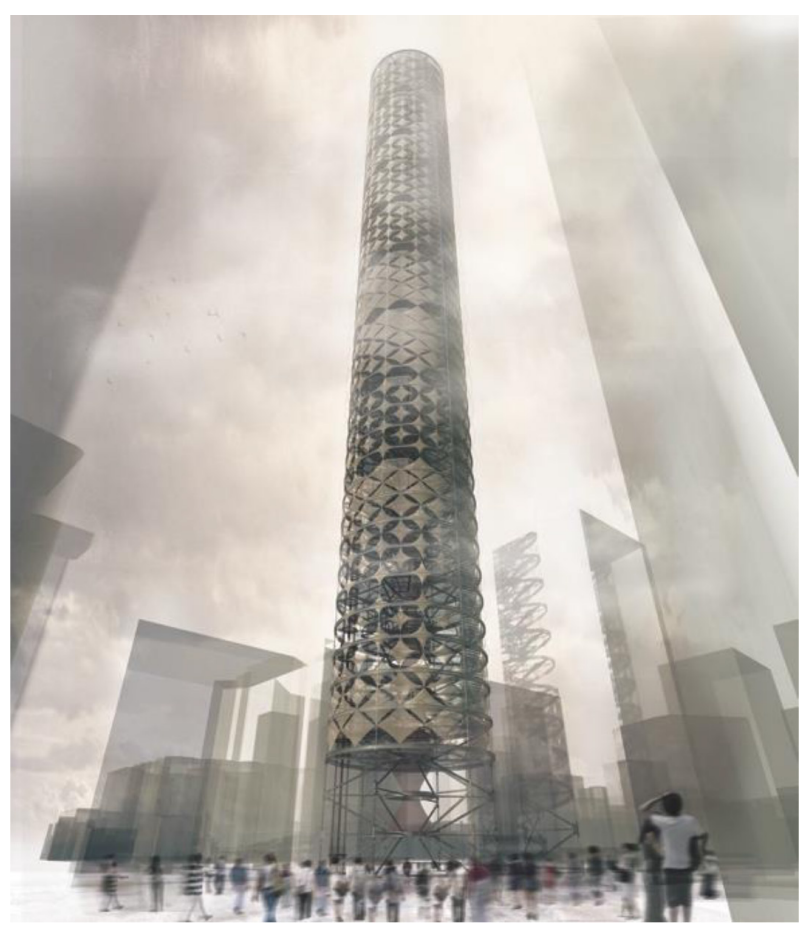

Figure 1: High-rise building with airflow core. 
inherent geometrical and mechanical features of modular assembly, reliability under differing heights and environmental conditions, re-usability and prefabrication. The functional program of public spaces and green unit areas over the height revives the concept of a vertical garden city within dense urban environments. A perspective of the proposed high-rise building concept with airflow core is presented in Fig. 1. The main principles of the airflow core are elaborated in section 3 of the paper.

The high-rise steel structure is principally an outrigger system with truss diagonals and vertical pretensioned cables at the outer perimeter [7]. The development of the high-rise kinetic structural mechanism that supports upward air movements follows a concept related to natural organisms' behaviour. In this frame, the jellyfish movement pattern is decoded, which is based upon creating pulses through vertical displacements and recovery processes, and applied on the structures kinematics and its materiality. The design proposes vertical PETG (polyethylene terephthalate glycol) lamellas interconnected with prestressed ETFE (ethylene tetrafluoroethylene) membranes, in utilizing the elements elastic residual capacity in bending, stretching and buckling to generate motion.

\section{PRIMARY STRUCTURE}

The high-rise structure has an overall height of $250 \mathrm{~m}$ and a diameter of $25 \mathrm{~m}$. The structure is divided over the height into units of $20 \mathrm{~m}$, each containing five functional levels. The primary structure consists of an outrigger system of six radial vertical trusses spanning between the core and the building perimeter, Fig. 2. Vertical cables are connected with the truss diagonals at the respective points at the perimeter of the structure. The horizontal levels are composed of three ring members, at the core, midspan and perimeter, and the radial beams. All axial beams are fixed connected to the ring members. Additional diagonals between the members increase the stiffness of the horizontal planes.

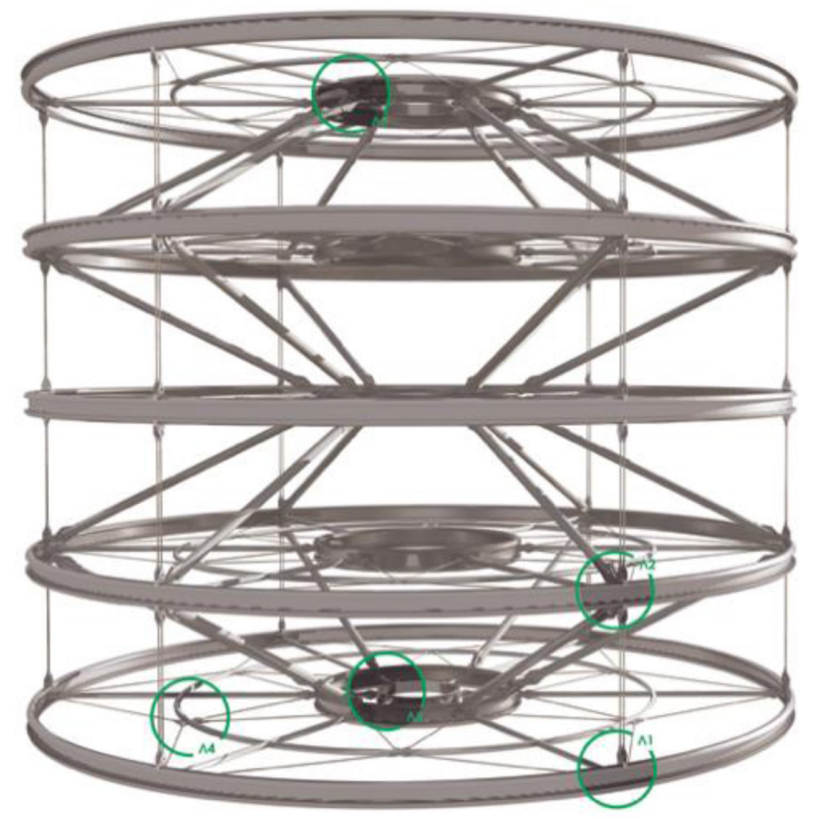

Figure 2: Primary structure unit. 
Table 1: Maximum absolute system responses for uniform vertical and horizontal loading (Axial force: N, Shear force: Q, Bending moment: M).

\begin{tabular}{lllll}
\hline System Responses & Diagonals & Ring Members & Radial Beams & Cables \\
\hline $\mathrm{N}[\mathrm{kN}]$ & 68638.63 & 58364.72 & 58958.63 & 33182.54 \\
{$[\mathrm{Q}[\mathrm{kN}]$} & - & 159.70 & 1349.35 & - \\
$\mathrm{M}[\mathrm{kNm}]$ & - & 2013.24 & 2715.63 & - \\
\hline
\end{tabular}

The dimensioning of the structural members has been based on Eurocode 3. A vertical load of $5 \mathrm{kN} / \mathrm{m}^{2}$ and a horizontal one of $1 \mathrm{kN} / \mathrm{m}^{2}$ have been considered for the analysis. The truss diagonals consist of round hollow sections of $36 / 1.5 \mathrm{~cm}$, the outer and middle ring members, of HEB450, and the inner ring members, of UPN320 sections. The vertical cable pairs have a diameter of $8 \mathrm{~cm}$.

\subsection{Structural behaviour}

The structural system has been investigated in its load-bearing behaviour with the FiniteElement software program SAP2000. In the nonlinear analysis conducted the cables have been initially modelled as frame objects with zero compression limit. The system develops primarily bending deformations, with an eigen-period of $3.97 \mathrm{~s}$. The maximum horizontal deformation of the structure amounts $29 \mathrm{~cm}$. The maximum absolute system responses for the uniform vertical and horizontal loading considered in the analysis are included in Table 1.

\section{SECONDARY KINETIC MECHANISM}

The core of the structure was envisaged to enhance the vertical air circulation through an enclosed kinetic mechanism. Its morphology investigation was based on the principles of aerodynamics, and the variation of the inner space diameter for increase of the air velocity, and avoidance of turbulence effects. From the area of biomimetic, the kinematics of jellyfish has been analysed and decoded for the development of the kinetic mechanism of the structure. The latter would contribute to an increase of the impulse of the airflow over the height. The jellyfish realises a radial contraction and expansion for its motion in water. Through contraction of its surface, it encloses water in the inside and following that, it pushes to the back through expansion of its surface for achievement of the impulse required, Fig. 3. Following this principle, the
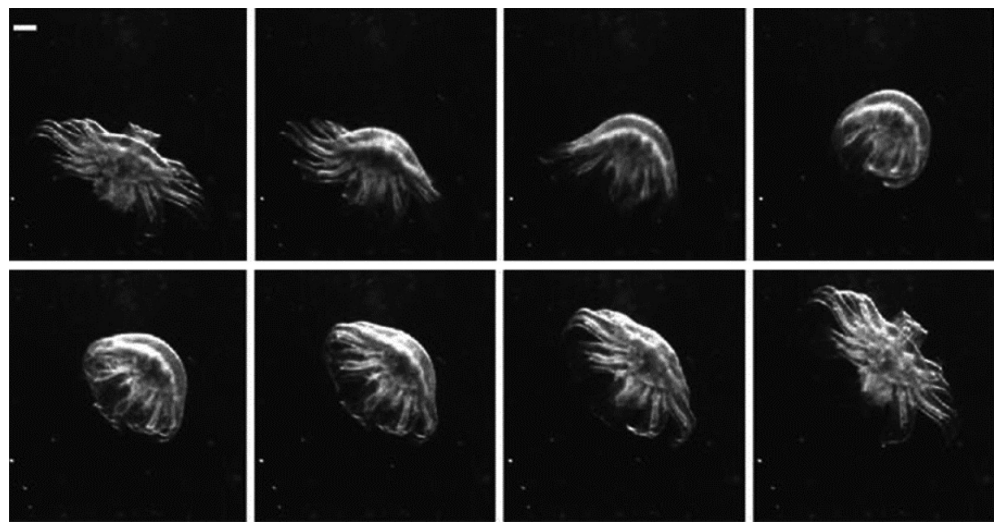

Figure 3: Jellyfish kinematics. 
surface elements of the mechanism are contracted and expanded for the required impulse development of the airflow. The mechanism units are divided into pairs of 'bubbles'. In a contraction bubble, incoming air is enclosed and temporarily stored. In an expansion bubble, the stored air is vertically channeled. The contraction process of the air suction bubble induces expansion of the subsequent bubble, i.e. inverse structural transformation, so that the air is further channelled in the inside, Fig. 4. Both bubbles act proportionally inversely. The surfaces are placed between three ring members. The ring members on both edges have constant diameter and absolute position, the middle one varies its position on the vertical axis, so that the process of transformation of each element during contraction and expansion is preserved.

The mechanism surface consists of bending-active PETG lamellas with $20 \mathrm{~m}$ length, 10-30 cm width and $10 \mathrm{~mm}$ thickness that are interconnected with prestressed ETFE membranes and supported by the ring members of UPN320 sections in the vertical axis. The lamellas are bended in their initial position. They are characterized by high elasticity, tension strength and residual stress behaviour enabling inverse transformations following uploading $[8,9]$. The latter is primarily utilized at the intermediate stage of motion, at the point that the bubble reaches its maximum level of contraction following the air input. At this stage, the elastic energy stored resets the element at the initial position, resulting in expansion of the bubble.

The oscillation of the elements and the development of impulses for the vertical airflow within the mechanism activate subsequent motions of the consecutive bubble units. The impulses developed may induce increase of the acceleration of the airflow with possible energy utilization. The building levels and the kinetic mechanism are placed at 5 to $25 \mathrm{~m}$ above ground, allowing the introduction of the airflow from the area below. The upward airflow is supported over the height through differentiation of the air pressure at the respective technical storeys of the building (chimney effect), Fig. 5. In addition, horizontal pumps are placed at these levels for the air suction.
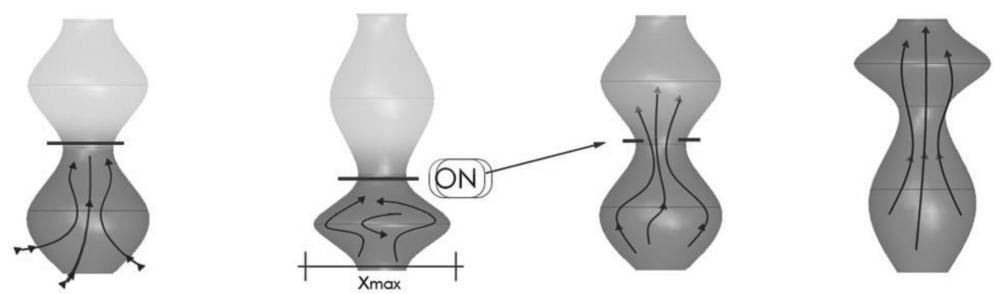

Figure 4: Kinetic mechanism bubbles unit transformability.
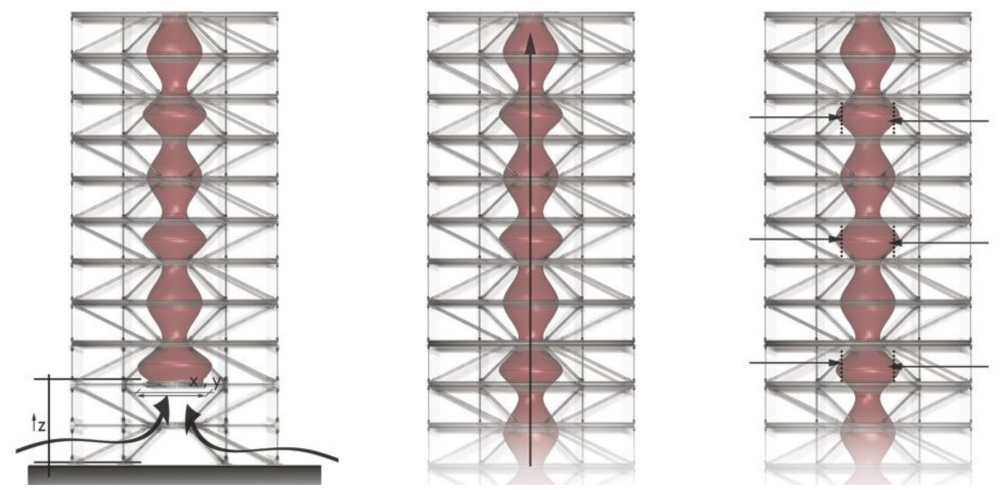

Figure 5: Vertical air ventilation concept. 


\subsection{Simulation analysis}

The digital simulation of the bending-active structure aims at providing a verification of the conceptual behaviour of the core mechanism, as well as a representation of the kinetic mechanism of the digital prototype using parametric design logic. This is considered to be a significant investigation, whereas the main concern is the kinetic structural behaviour, induced by the behaviour of the elastic materials of the structural members. While focusing on the examination of the structural behaviour, parametric associative tools are used during the process of simulation, which allows an investigation at the conceptual design level and provides initial insight into the kinetic behaviour of the structure with regard to its geometrical transformation stages and conceptual physical behaviour. The analysis outcomes make up an integral part of the structural performance verification, where advanced structural analysis software is used and numerical results are obtained. For the preliminary simulation of the kinetic structure, the parametric design software Grasshopper and the live physics engine software Kangaroo (plug-in for Rhino and Grasshopper respectively) were used.

During the simulation process, the initial geometrical form, the parametric associative control, as well as the physical properties of the overall structural materiality were taken into consideration. In terms of geometrical and physics-based parametric control and behaviour, the structure was initially examined through isolation of one pair of bubble units focusing on the curvilinear bending-active stripes interconnected with prestressed ETFE membranes, and then the structural system as a whole (synthesis of many pairs).

The structure was examined by classifying its elements into two main categories, the PETG elastic lamellas acting as bending-active members and the prestressed ETFE membranes that provide stabilization and control of any possible deformations of the interconnected elastic units. These are associatively related and physically encoded based on forces including bending, pretension, gravity (self-loading), spring force and vertical rising wind flow.

The PETG lamellas and their elasticity were digitally simulated using bending-active behaviour rules. The ETFE membrane was also digitally simulated, keeping the bending elements together, while simultaneously maintaining the ability of its shape to be altered, due to its elastic properties. Analytically, the procedure involves the design definition of curved shape stripes with a thinner cross section at both ends, as well as in the middle, Fig. 6. To reach this outcome, a parametric division was created, into which two imaginary lines were designed to connect points and turn them into segments, in order to produce the 2D curved stripe. At the end of each line that represents the points of connection, the membrane consists of segments that are created in the empty space between the stripes. The deformed curved shape appears in two and three dimensions, representing the kinematically active position of the whole system. Initially, this is achieved by the trend of the curved stripes to be aligned in a straight line using any force or pressure. In order to undergo a respective bending alteration and keep the stripes in the initial bending active position, their edges are anchored to a fixed-section ring, while the points in the middle are joined to a smaller fixed-section ring, providing the possibility of vertical movement. The two rings at the ends are positioned in such a way that the distance between them is smaller than the length of the stripe in straight position. Thereby, the last two rings, which are positioned at the edges of the bubble pair units, place the whole system in an initial compression position, and enable energy to be stored in the elastic material of the bending stipes. Consequently, the system is already placed in an active position, which is controlled and simulated by applying spring behaviour on the basis of Hooke's Law of elastic stress-strain behaviour [10]. Therefore, with regard to the force or pressure applied to these elastic members, there is a proportional distortion with 


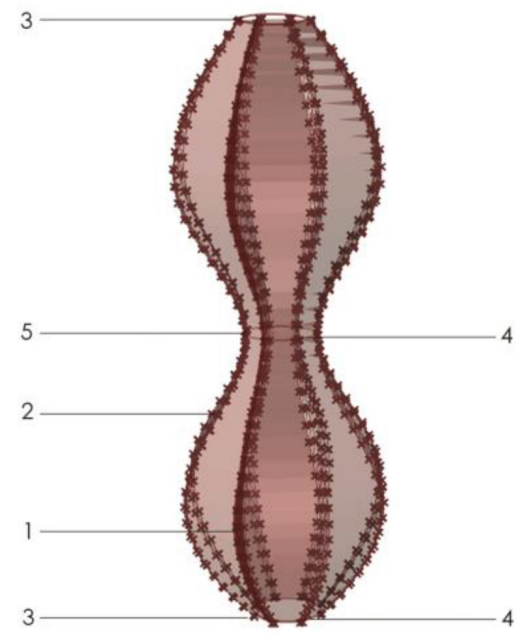

\author{
1. Curved shape stripe \\ 2. Points \\ 3. Ancored points \\ 4. Fixed section ring \\ 5. Joined points
}

Figure 6: Digital representation of structural components representing a single bubble's structure.

the help of the middle ring that moves vertically. When it reaches the maximum amount of distortion, the energy stored is used to reset the system in the initial shape and position.

The simulation of the stripe members' behaviour is achieved by applying bending and spring forces, while the physical behaviour of the membrane is simulated using spring and wind forces. An external parameter of wind force is applied. When this enters the interior of the structure, it creates a distortion of the area surrounded by the stripes and the membrane, which causes the vertical movement impulsion of the stripes. This behaviour expands the lower bubble inner area, creating a respective contraction of the upper inner region. As a consequence, the stored energy due to the structural materiality, works as the actuator of the system, forcing the shape to reach a recovery position, through the method of pulse, Fig. 7.

Consequently, the investigation procedure enables decoding of the material in view of the members' interaction behaviour with different environments. In the case example examined, structural deformation is influenced by external forces, due to vertical upward wind flow and self-loading conditions. This simulation process provided a series of system deformation scenarios based on motion frequency and shape alteration, induced by the wind speed. The result represents the possible behaviour of the prototype under the use of force or pressure. Therefore, through the process of digitization and simulation, a relation between modelling and realization was created.
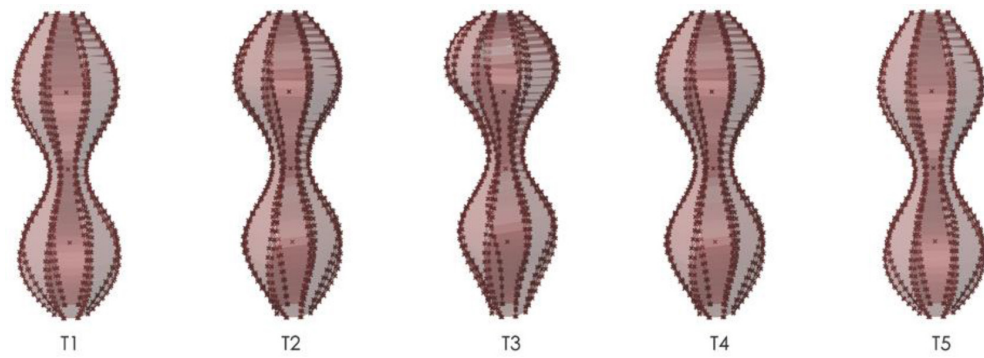

Figure 7: Simulation steps indicating the transformability of a bubble unit. 


\section{CONCLUSIONS}

A high-rise airflow structural concept based on an incorporated kinetic mechanism has been presented in the current paper. The concept development aims at fostering sustainability in contemporary dense urban environments through enhancement of airflow over the height of high-rise buildings. The integration of the kinetic mechanism in hybrid outrigger high-rise structures is based on a design syntax of modular assembly, prefabrication of the components and adaptability for different building heights. The kinetic mechanism is derived from the area of biomimetic following a top-down approach. The jellyfish movement pattern provided the kinematics of the mechanism, realized by units of transformable bubble pairs arranged vertically within the structures core. The bubbles consist of bending-active members interconnected with prestressed membranes and ring members. Utilization of the elastic material properties of the members enables sequential motions through contraction and expansion of the bubbles in support of the vertical airflow. For verification and evaluation of the airflow concept and the kinetic structure proposed, further wind-tunnel experiments are necessary in view of both, the core morphology and the proposed kinematics.

\section{REFERENCES}

[1] Newman, P., Sustainability and cities: the role of tall buildings in the new global Agenda. Proceedings of the CTBUH Sixth World Congress, Melbourne, Australia, pp. 76-109, 2001. http://dx.doi.org/10.4324/noe0415232418.ch4

[2] Castillo, P. \& Beyers, M., New solar initiatives in supertall buildings: the spire at Ras Al Khaimah. CTBUH Council on Tall Building and Urban Habital Journal, 3, pp. 20-27, 2010.

[3] Land, P., Innovations in sustainability at height: experimental tall buildings. CTBUH Council on Tall Building and Urban Habital 8th World Congress, pp. 1-10, 2008.

[4] Smith, R.F. \& Killa, S., Bahrain World Center (BWTC): the first large-scale integration of wind turbines in a building. The Structural Design of Tall and Special Buildings, 16, pp. 429-439, 2007.

http://dx.doi.org/10.1002/tal.416

[5] Lu, L. \& Ip, K.Y., Investigation on the feasibility and enhancement methods of wind power utilization in high-rise buildings of Hong Kong. Renewable and Sustainable Energy Reviews, 13, pp. 450-461, 2009. http://dx.doi.org/10.1016/j.rser.2007.11.013

[6] Sharpe, T. \& Proven, G., Crossflex: concept and early development of a true building integrated wind turbine. Energy and Buildings, 42, pp. 2365-2375, 2010. http://dx.doi.org/10.1016/j.enbuild.2010.07.032

[7] Choi, H., Ho, G., Joseph, L. \& Mathias, N., Outrigger Design for High-Rise Buildings. An Output of the CTBUH Outrigger Working Group. Council on Tall Buildings and Urban Habitat, Chicago, 2012.

[8] Knippers, J. \& Speck, T., Design and construction principles in nature and architecture. Bioinspiration \& Biomimetics, 7, 2012 Online, available at: http://stacks.iop.org/ $\mathrm{BB} / 7 / 015002$

[9] Phocas, M.C., Kontovourkis, O. \& Nicolaou, N., Design concept of a kinetic formactive hybrid envelope structure. Design \& Nature and Ecodynamics, 9(1), pp. 13-30, 2014.

http://dx.doi.org/10.2495/DNE-V9-N1-13-30 
184 M.C. Phocas, et al., Int. J. of Design \& Nature and Ecodynamics. Vol. 12, No. 2 (2017)

[10] Ahlquist, S. \& Menges, A., Realizing formal and functional complexity for structurally dynamic systems in rapid computational means: computational methodology based on particle systems for complex tension-active form generation. Advances in Architectural Geometry 2010, eds C. Ceccato, L. Hesselgren, M. Pauly, H. Pottmann \& J. Wallner, Springer: Vienna, pp. 205-220, 2011. 